\title{
ARTÍCULOS
}

\author{
Sometido 16.03.2020. Aprobado 26.08.2020.
}

Evaluado por el sistema double blind review. Editor Científico: Mauricio Reinert

Versión original | DOI: http://dx.doi.org/10.1590/So034-759020210406

\section{EL EFECTO DE LA DIVERSIDAD DE SOCIOS DE RELACIONES INTERORGANIZACIONALES EN LA INNOVACIÓN DE PRODUCTOS}

\section{Efeito da diversidade de parceiros de relações interorganizacionais na inovação de produtos The effect of the diversity of interorganizational partners on product innovation}

Ángeles Montoro-Sánchez ${ }^{1}$ | mangeles@ccee.ucm.es | ORCID: 0000-0003-0575-4568

Youseline Garavito-Hernández² | ygaravito2@udi.edu.co | ORCID: 00oo-0002-1644-9959

Ana M. Romero-Martínez³ | amromero@ucm.es | ORCID: 00oo-0001-7419-554X

1 Universidad Complutense de Madrid, Facultad de Ciencias Económicas y Empresariales, Departamento de Organización de Empresas y Marketing,
Madrid, España
2 Universidad de Investigación y Desarrollo, Facultad de Ciencias Administrativas, Económicas y Contables, Bucaramanga, Santander, Colombia
${ }^{3}$ Universidad Complutense de Madrid, Facultad de Ciencias Económicas y Empresariales, Departamento de Organización de Empresas y Marketing,
Madrid, España

\section{RESUMEN}

El objetivo de este estudio es analizar el impacto de la diversidad del tipo de socios en las relaciones interorganizacionales en la innovación de productos. La contrastación empírica con datos del Panel de Innovación Tecnológica de España muestra que la diversidad de tipos de socios en las relaciones interorganizacionales influye positivamente en la innovación de productos. En concreto, se pueden obtener mayores beneficios en la innovación de bienes cuando hay diversidad de relaciones entre socios comerciales (clientes y proveedores); y en la innovación de servicios con diversidad de relaciones entre socios no industriales (consultores, universidades y entidades de investigación). Finalmente, los resultados no permiten afirmar que demasiada diversidad produce rendimientos decrecientes en la innovación.

PALABRAS CLAVE | Innovación de producto, diversidad, relaciones interorganizacionales, innovación de bienes, innovación de servicios.

\section{RESUMO}

O objetivo deste estudo é analisar o impacto da diversidade do tipo de parceiros nas relações interorganizacionais em inovação de produtos. A aplicação empírica com dados do Painel de Inovação Tecnológica da Espanha mostra que a diversidade de tipos de parceiros nas relações interorganizacionais influencia positivamente a inovação de produtos. Em particular, pode-se ter maiores benefícios para a inovação em bens quando existem relações diversas entre parceiros comerciais (clientes e fornecedores); e inovação em serviços quando a diversidade de relacionamentos ocorre entre parceiros não industriais (consultores, universidades e entidades de pesquisa). Finalmente, os resultados não permitem afirmar que muita diversidade produz retornos decrescentes de inovação.

PALAVRAS-CHAVE I inovação de produtos, diversidade, relacionamentos interorganizacionais, inovação de bens, inovação de serviços.

ABSTRACT

This paper analyzes the impact on product innovation of different types of interorganizational relationship partners. An empirical contrast with data taken from the Spanish Technological Innovation Panel (based on Eurostat's Community Innovation Survey) shows that diversity in the types of partners involved in interorganizational relationships has a positive effect on product innovation. In particular, increased benefits can be achieved in the innovation of goods when there is diversity in the relationships that exist between business partners (customers and suppliers); and in service innovation when there is diversity in the relationships between non-industrial partners (consultants, universities and research entities). Finally, given the results it is not possible to state that a lot of diversity reduces the returns on innovation.

KEYWORDS I Product innovation, diversity, interorganizational relationships, goods innovation, service innovation. 


\section{INTRODUCCIÓN}

Para sobreviviry ser competitivas las empresas deben innovar (Belderbos, Gilsing, Lokshin, Carree, \& Sastre, 2018). Como no todas disponen internamente de los recursos, capacidades y conocimientos para hacerlo (Schoenmakers \& Duysters, 2006), recurren a las relaciones interorganizacionales (RI) para adquirirlos (Beers \& Zand, 2014; Belderbos et al., 2018; Das \& Teng, 2000). Las RI son acuerdos de colaboración que la empresa mantiene con diferentes socios estratégicos para intercambiar o compartir recursos. La literatura muestra que tienen un efecto positivo y significativo en la innovación de productos (IP) pues permiten a las empresas combinar y complementar sus recursos y capacidades con los de sus socios (Faems, Looy, \& Debackere, 2005). De ahí que exista un creciente interés por estudiar las RI y su impacto en la IP (Belderbos, Carree, Lokshin, \& Sastre, 2015; Silva \& Leitão, 2009).

Para innovar las empresas necesitan cooperar con diferentes tipos de socios como son los clientes, proveedores, competidores, empresas del mismo grupo e instituciones de investigación para alcanzar diferentes objetivos a través de la transferencia de diversos conocimientos (Arranz \& Arroyabe, 2008). Ahora bien, cada tipo de socio contribuye de forma diferente a la innovación (Belderbos, Carree, \& Lokshin, 2004; Bogers, Afuah, \& Bastian, 2010; Leeuw, Lokshin, \& Duvsters, 2014; Nieto \& Santamaría, 2007; Story, O’Malley, \& Hart, 2011). La literatura reciente destaca la importancia de colaborar con distintos tipos de socios y la gestión de la cartera de alianzas de la empresa (Hagedoorn, Lokshin, \& Zobel, 2018). Ahora bien, la mayoría de los estudios previos se centran en lo diversos que son los socios mirando hacia la variedad o amplitud de socios por el número diferente de estos (Belderbos et al., 2018; Kobarg, Stumpf-Wollersheimb, \& Welpe, 2019; Nieto \& Santamaría, 2007). Sin embargo, apenas se ha analizado la diversidad entre estos socios con una perspectiva de heterogeneidad entre ellos.

En este estudio, la diversidad de las RI hace referencia al grado de heterogeneidad entre los diferentes tipos de socios estratégicos con los que las empresas se relacionan (Hagedoorn et al., 2018). Dicha heterogeneidad depende de las diferencias entre los socios derivadas de que la relación sea de carácter vertical u horizontal, competitiva o complementaria, o en función del objetivo que lleve a la empresa a relacionarse con cada tipo de socio (Beck \& Schenker-Wicki, 2014; Beers \& Zand, 2014; Belderbos et al, 2018). Por ello, los tipos de socios con los que la empresa coopera es un factor clave para evaluar el efecto de la diversidad de RI en la innovación (Lee, Kirkpatrick-Husk, \& Madhavan, 2017; Nieto \& Santamaría, 2010). Ahora bien, la evidencia empírica, además de escasa, no es concluyente (Hagedoorn et al., 2018; Lee et al., 2017). Algunos estudios encuentran que el grado de diversidad de tipos de socios influye positivamente en la innovación, mientras que en otros es negativa, o no lineal (Beck \& Schenker-Wicki, 2014; Beers \& Zand, 2014; Kobarg et al., 2019; Leeuw et al., 2014; Meyskens \& Carssrud, 2013).

Ante la importancia de la cooperación con diferentes tipos de socios en la innovación de productos (Hagedoorn et al., 2018) y la necesidad de proporcionar conocimiento más específico y evidencia empírica del efecto de la diversidad de estos socios sobre la innovación, el objetivo de este trabajo es analizar el impacto de la diversidad de tipos de socios de las RI en la IP. Para ello, la principal novedad que implica este trabajo es, en primer lugar, el estudio del efecto de la diversidad de RI en la IP respecto a la heterogeneidad entre los diferentes tipos de socios y la propuesta de tres grupos de socios estratégicos: socios no industriales (instituciones de investigación y consultores), socios industriales (empresas del mismo grupo y competidores) y socios comerciales (clientes-proveedores) (Ardito, Petruzzelli, \& Albino, 2015; Silva \& Leitão, 2009). Las empresas eligen sus socios estratégicos dependiendo del tipo de conocimiento que desean adquirir, y cada uno de estos grupos de socios 
aportan conocimientos diferenciadores a la IP (Ardito et al., 2015; Story et al., 2011; Wagner \& Hoegl, 2006). Con este análisis del impacto de la diversidad de los diferentes tipos de socios estratégicos en la IP este trabajo aporta fundamentación y evidencia a la literatura sobre diversidad de RI ante la ausencia de resultados concluyentes (Hagedoorn et al., 2018; Lee et al., 2017). En segundo lugar, se estudia no solo la relación lineal sobre la innovación, sino además una relación en forma de U invertida entre la diversidad de RI y la innovación para cada uno de los grupos de socios estratégicos (Beck \& Schenker-Wicki, 2014; Kobarg et al., 2019; Leeuw et al., 2014). Esto es, aunque la diversidad de RI es favorable para la innovación, a medida que esta aumenta la empresa podría no obtener los resultados positivos esperados (Hagedoorn et al., 2018; Lee et al., 2017). Finalmente, el estudio aporta nueva evidencia empírica al emplear el Panel de Innovación Tecnológica de España.

Para alcanzar el objetivo de investigación, primero se presenta la revisión de la literatura. Después, la metodología y medida de las variables. Por último, los resultados y las conclusiones, futuras líneas de investigación y limitaciones.

\section{MARCO TEÓRICO E HIPÓTESIS DE TRABAJO}

Una importante fuente de ventaja competitiva es la capacidad de la empresa de crear y mantener RI valiosas (Lavie, 2006), para acceder a conocimientos de los socios para mejorar sus actividades innovadoras y desarrollar nuevos productos y servicios (Das \& Teng, 2000; Martinez-Noya \& Narula, 2018; Miotti \& Sachwald, 2003; Schoenmakers \& Duysters, 2006).

La IP es una actividad intensiva en conocimiento que requiere adquirirlo fuera de la empresa (Ozer \& Zhang, 2015). Si la empresa se relaciona con diversos tipos de socios estratégicos (clientes, proveedores, competidores e instituciones de investigación) podrá obtener conocimientos, información y sinergias nuevos y complementarios (Beers \& Zand, 2014; Nieto \& Santamaría, 2007) que favorezcan el aprendizaje y desarrollo de nuevos productos y su introducción en el mercado (Kobarg et al., 2019; Lavie, 2009). Este acceso a más conocimientos, por la diversidad de socios con los que colabora, provee a la empresa de habilidades para innovar y mejorar su ventaja competitiva (Beck \& Schenker-Wicki, 2014; Burg, Berends, \& Raaij, 2014; Kobarg et al., 2019; Lee et al., 2017; Meyskens \& Carssrud, 2013). Por el contrario, cuando hay poca diversidad y las interrelaciones son siempre con los mismos socios, el isomorfismo reduce el acceso a nuevas oportunidades (Uzzi, 1997). Por lo tanto, con la hipótesis 1 proponemos que el grado de diversidad de socios con los que la empresa colabora (Beers \& Zand, 2014; Duysters \& Lokshin, 2011; Jiang, Qingjiu, \& Santoro, 2010) afectará positivamente a la innovación.

H1: La diversidad de socios en las RI favorece la IP.

El tipo de socio tiene gran relevancia (Nieto \& Santamaría, 2010; Tether, 2002). La empresa busca beneficiarse de la diversidad escogiendo aquellos socios que más le faciliten innovar (Leeuw et al., 2014). Así, eligen socios estratégicos en función de factores (Miotti \& Sachwald, 2003; Tether, 2002) tales como la complementariedad de conocimientos y habilidades (Mowery, Oxley, \& Silverman, 1996), la experiencia previa (Wagner \& Hoegl, 2006), la compatibilidad estratégica -afinidad de objetivos estratégicos y competitivos- y la compatibilidad organizativa -similitud en estilos de dirección, cultura, etc.- (Emden, Calantone, \& Droge, 2006). Por ello, la literatura clasifica los tipos de socios en tres categorías: socios comerciales (clientes y proveedores), socios industriales (competidores y empresas del mismo grupo), y socios no industriales (consultoras, universidades y 
otras instituciones de investigación) (Ardito et al., 2015; Arranz \& Arroyabe, 2008; Belderbos et al., 2018; Silva \& Leitão, 2009). Cada uno de ellos aportan conocimientos diferentes a la IP (Ardito et al., 2015; Story et al., 2011; Wagner \& Hoegl, 2006). La literatura previa solo ha estudiado el efecto de cooperar con cada uno de ellos de forma individual o en los grupos anteriormente señalados en la innovación (Nieto \& Santamaría, 2007). Nuestro objetivo es investigar cómo el grado de diversidad en cada grupo afecta a la IP.

La literatura previa reconoce que las RI con los socios comerciales (clientes y proveedores) (Mowery et al., 1996) facilitan el acceso a nuevas tecnologías, experiencias y conocimientos; necesarios para desarrollar nuevos productos (Bogers et al., 2010; Romijn \& Albaladejo, 2002; Tether, 2002). Los clientes pueden ofrecer ideas sobre nuevas tendencias en el mercado y posibles aplicaciones de nuevos productos (Burg et al., 2014; Ozer \& Zhang, 2015). La relación con los clientes favorece la IP pues la empresa obtiene mayor información para satisfacer mejor sus gustos y necesidades (Miotti \& Sachwald, 2003), especialmente si estos productos son novedosos para el mercado (Ashok, Narula \& Martínez-Noya, 2016; Belderbos et al., 2004; Nieto \& Santamaría, 2007; Tether, 2002). Por otra parte, las relaciones con proveedores pueden resultar beneficiosas porque ofrecen conocimiento operativo y acceso a activos especializados y complementarios que ayudan a mejorar la calidad y eficiencia del producto, proteger tecnologías o diseños novedosos, mejorar los procesos productivos y reducir costes y riesgos (Burg et al., 2014; Faems et al., 2005; Ledwith \& Coughlan, 2005; Miotti \& Sachwald, 2003; Nieto \& Santamaría, 2007; Ozer \& Zhang, 2015). Igualmente, mantener relaciones con ambos socios estratégicos permite a las empresas comercializar de forma más eficiente y rápida sus nuevos productos para aumentar sus ventas y realizar nuevas innovaciones (Rosenzweig, Roth, \& Dean, 2003). La hipótesis analiza el efecto positivo de la diversidad de mantener RI con socios comerciales (clientes y proveedores) en la innovación:

H1a: La diversidad de las RI con clientes y proveedores favorece la IP.

En cuanto a aquellos socios con los que se comparte algún vínculo corporativo (empresas del mismo grupo), o competitivo, como los competidores, la diversidad de RI es beneficiosa porque permite compartir la incertidumbre y los costes y riesgos de la investigación y acceder más fácilmente a activos, recursos y conocimientos complementarios necesarios (Belderbos et al., 2004; Burg et al., 2014; Emden et al., 2006; Nieto \& Santamaría, 2007) para posicionarse mejor en el mercado (Ardito et al., 2015; Belderbos et al., 2018). De los rivales se puede aprender sobre las características, diseño y comercialización de nuevos productos alternativos (Ozer \& Zhang, 2015). Cooperar con empresas del mismo grupo ayuda con información complementaria del mismo sector o sectores afines cuando la relación es de tipo complementario y horizontal (Belderbos et al., 2004; Tether, 2002). Con la hipótesis $1 \mathrm{~b}$ exploramos el efecto positivo de esta diversidad de relaciones:

H1b: La diversidad de las RI con empresas del mismo grupo y competidores favorece la IP.

Por último, están aquellos socios que proveen a la empresa de conocimientos que no pueden adquirir en el mercado ni a través de la competencia o empresas del mismo grupo, estos son: consultores, universidades, organismos públicos de investigación y centros tecnológicos (socios no industriales) (Mowery et al., 1996; Wagner \& Hoegl, 2006). Estos socios contribuyen a la innovación aportando conocimientos científicos y asesoría que favorece la creación de nuevas ideas y la mejora de actividades de I+D (Dowling \& Helm, 2006; Frenz \& letto-Gillies, 2009). Así, las RI con consultores ayudan a compartir experiencias, definir necesidades concretas en innovación y aportar ideas novedosas y complementarias (Bessant \& Rush, 1995; Bruce, Leverick, Littler, \& Wilson, 1995); y las relaciones con universidades y centros de investigación permiten el acceso a conocimientos científicos, equipos técnicos con personal clave para las innovaciones, como profesores o investigadores, o 
nuevas opciones tecnológicas, que favorecen el desarrollo de nuevos productos (Frenz \& letto-Gillies, 2009). Con la hipótesis 1c exploramos el efecto positivo de la diversidad de RI con socios no industriales.

H1c: La diversidad de las RI con consultores, universidades, organismos públicos de investigación y centros tecnológicos favorece la IP.

Ahora bien, no toda la evidencia previa muestra una relación positiva entre diversidad e innovación (Kobarg et al., 2019; Luo, 2002; Nieto \& Santamaría, 2010). Existe evidencia previa sobre esta relación sin resultados concluyentes (Beck \& Schenker-Wicki, 2014; Meyskens \& Carssrud, 2013). Algunos autores sostienen que la relación tiene forma de U invertida (Beck \& Schenker-Wicki, 2014; Duysters \& Lokshin, 2011; Haans, Pieters, \& Zi-Lin, 2016; Kobarg et al., 2019; Laursen \& Salter, 2006; Leeuw et al., 2014; Vasudeva \& Anand, 2011). Esto es porque las empresas necesitan recursos internos para explotar los conocimientos adquiridos de los diferentes socios estratégicos (Faems, Janssens, Madhok, \& Looy, 2008). Sin ellos es difícil implantar los conocimientos adquiridos (Leeuw et al., 2014). Además, su explotación puede generar problemas y costes de coordinación por la complejidad de gestionar conocimientos diversos (Hagedoorn et al., 2018; Jiang et al., 2010; Wuyts \& Dutta, 2014). También las empresas pueden contar con esos recursos pero que no les compense explotarlos por la relación coste-beneficio o porque la entrada de información con nuevas ideas no se produce en el momento adecuado (Chen, Chen, \& Vanhaverbeke, 2011; Knudsen \& Mortensen, 2011; Nieto \& Santamaría, 2007). Para que la IP tenga éxito es necesario integrar e implantar en la empresa ambos conocimientos (externo e interno) interrelacionados (Ozer \& Zhang, 2015). Otros motivos para el efecto en forma de U invertida de la diversidad de RI en la IP es la falta de control sobre el intercambio de información o de confianza entre los socios. Esto puede propiciar comportamientos oportunistas (Santamaría, Nieto, \& Miles, 2012) y spillovers de conocimiento técnico crítico (Burg et al., 2014) afectando a la eficacia de la diversidad de las RI para adquirir conocimientos relevantes para el desarrollo de innovación (Das \& Teng, 2000). Con la hipótesis 2 exploramos si el efecto de la diversidad de socios en las RI sigue una relación en forma de $U$ invertida:

H2: El efecto de la diversidad de RI en la IP sigue una relación en forma de U invertida.

Ahora bien, si nos centramos en los cada uno de los grupos de socios estratégicos, existen pocos estudios que contrasten si el efecto de la diversidad de RI en la IP sigue una relación en forma de U invertida (Hagedoorn et al., 2018; Martinez-Noya \& Narula, 2018) en cada uno de estos grupos (Ardito et al., 2015).

Aunque la mayoría de los autores concluyen que la diversidad de relaciones entre los socios comerciales permite a las empresas acceder a experiencias y conocimientos más amplios y diversos necesarios para crear nuevos modelos, mejorar los diseños existentes y desarrollar nuevos productos (Bogers et al., 2010; Romijn \& Albaladejo, 2002), a medida que aumenta la diversidad de relaciones entre los socios comerciales, la explotación de estos recursos en la empresa puede ser difícil, ya que cuanto más diversos sean los conocimientos adquiridos mayor será la dificultad para coordinarlos e integrarlos. Por tanto, los beneficios de mantener una diversidad de relaciones con socios comerciales pueden verse reducidos (Jiang et al., 2010; Wuyts \& Dutta, 2014). La hipótesis 2 a explora el efecto de la diversidad de relaciones con socios comerciales sobre la innovación positiva creciente hasta alcanzar un punto en el que empieza a disminuir.

H2a: El efecto de la diversidad de RI con clientes y proveedores en la IP sigue una relación en forma de U invertida.

En cuanto a los socios industriales, aunque la diversidad de relaciones con estos permite a las empresas acceder más fácilmente a conocimientos complementarios para innovar (Belderbos et al., 2004; Emden 
et al., 2006; Nieto \& Santamaría, 2007), al aumentar la diversidad de relaciones puede ser más arriesgada. El conocimiento entre los socios puede ser redundante, surgir desconfianza entre los socios, comportamientos oportunistas o incluso la apropiación indebida de capacidades. Por ello, la cooperación puede ser poco estable o incluso disolverse y no generar el efecto positivo para desarrollar nuevos productos (Dussauge, Garrette, \& Mitchell, 2000; Gimeno, 2004; Monteiro, Mol, \& Birkinshaw, 2017). Con la hipótesis 2b exploramos el efecto de la diversidad de relaciones con socios industriales sobre la innovación positiva creciente hasta alcanzar un punto en el que empieza a disminuir.

Hzb: El efecto de la diversidad de RI con empresas del mismo grupo y competidores en la IP sigue una relación en forma de $U$ invertida.

Por último, la literatura reconoce que la diversidad de relaciones con socios no industriales, como consultores, universidades y entidades de investigación contribuye a la IP aportando conocimientos que favorecen la mejora continua en las actividades de I+D (Frenz \& letto-Gillies, 2009). Sin embargo, el aumento de conocimientos externos resultado de este tipo de diversidad de RI puede generar actividades de I+D que no encajen con las estrategias de investigación de la empresa (Numprasertchai \& Igel, 2005) por lo que no tendrán el impacto positivo esperado en la IP. El éxito de la diversidad de relaciones con socios no industriales está en identificar hasta qué punto esta diversidad es favorable. La hipótesis 2 c explora si el efecto de la diversidad de relaciones con socios no industriales sobre la innovación es positivo y creciente hasta alcanzar un punto en el que empieza a disminuir.

H2c: El efecto de la diversidad de RI con consultores, universidades y entidades de investigación en la IP sigue una relación en forma de U invertida.

La figura 1 muestra gráficamente todas las hipótesis del trabajo.

\section{Figura 1. Hipótesis de investigación}

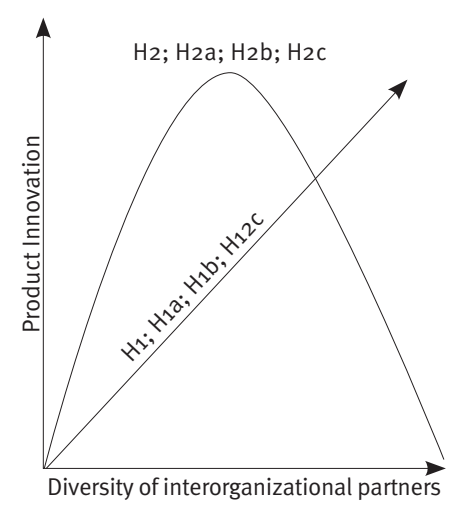

\section{METODOLOGÍA}

\section{Población y muestra}

La fuente de información utilizada en este trabajo ha sido el Panel de Innovación Tecnológica de España (Community Innovation Survey de Eurostat,) elaborado por el Instituto Nacional de Estadística. Se han considerado 
los datos de 2011 y 2012 con el fin de tomar los valores de innovación del periodo 2010-2012 y de cooperación del periodo 2009-2011 (años con datos de acceso abierto y gratuito). Para las variables independientes se han empleado los valores del 2011, mientras que para la variable dependiente se han utilizado los del 2012. Con relación a las variables de control, para los gastos de innovación internos y externos en I+D se utilizaron los valores del 2011 y para las variables tamaño, antigüedad y sector, los datos del 2012.

Respecto a la selección de la muestra se tuvieron en cuenta tres criterios: que las empresas estuviesen operativas, que tuvieran más de 10 empleados y que habiendo respondido la pregunta de cooperación con otras empresas, hubieran cooperado por lo menos con un tipo de socio estratégico. La muestra final estuvo conformada por 2141 empresas. El 34,89\% tiene menos de 20 años de antigüedad y el 65,11\%, más de 20 años. Según la recomendación de la Comisión Europea de 6 de mayo de 2003 respecto al número de empleados, se observa que el 33,49\% son empresas pequeñas (10-49 empleados), el 38,81\% medianas (50-249 empleados) y el 27,70 $\%$ grandes empresas (más de 250 empleados). El 62,21 \% de la muestra son empresas manufactureras y el 37,78 $\%$, de servicios. Respecto a la innovación, el $67 \%$ del total de empresas realiza IP (1433 empresas), de las cuales el $56 \%$ (1280 empresas de 2141) innovan en bienes y el $33 \%$ (713 empresas), en servicios.

\section{Medición de las variables}

La IP consiste en el desarrollo de nuevos bienes o servicios. De acuerdo con el Panel de Innovación Tecnológica la IP (bien o servicio) supone la comercialización de un producto nuevo o mejorado significativamente con respecto a sus capacidades, facilidad de uso, componentes o subsistemas. Las innovaciones de productos (nuevas o mejoradas) deben ser nuevas para la empresa, pero no tienen por qué ser nuevas para el mercado. La IP se suele separar entre innovación de bienes (IB) (un bien es generalmente un objeto tangible, como un teléfono inteligente, muebles o software empaquetado, pero el software descargable, la música y la película también son bienes, e innovación de servicios (IS) (un servicio suele ser intangible, como venta minorista, seguros, cursos educativos, viajes aéreos, consultoría, etc.). De acuerdo a la información del Panel y las medidas de innovación utilizadas en estudios previos (Simonen \& McCann, 2008) hemos empleado tres variables dicotómicas que toman valor 1 si la empresa ha innovado. En primer lugar, una medida que representa la IP como medida única global, y en segundo lugar, dos indicadores que representan la IB y la IS.

Respecto a las variables independientes, para medir la diversidad de tipos de socios en las RI hemos empleado el índice de Blau. Este está considerado como el índice más fiable y consistente de heterogeneidad para medir la diversidad de RI (Meyskens \& Carsrud, 2013). Se calcula como $B=1-\sum_{i=1}^{k}(p i)^{2}$, donde B es el índice de Blau y p es el porcentaje de miembros en cada i-ésima categoría de las k existentes. Cuanto más elevado es el valor de B, mayor será el grado de diversidad, los valores de B varían entre o y $(k-1) / k$. De este modo hemos calculado la diversidad total y la diversidad para cada uno de los tres grupos de socios considerados en el marco teórico (Arranz \& Arroyabe, 2008).

En la diversidad de tipo de socios total, el número de categorías es 8 ya que consideramos las siguientes alternativas de RI: clientes, proveedores, competidores, empresas del mismo grupo, consultores, universidades, organismos públicos de investigación y centros tecnológicos (Beck \& Schenker-Wicki, 2014). Así, siendo K=8 para la variable diversidad de RI, la diversidad máxima será cuando B alcance el valor de o,875.

En la diversidad de tipos de socios para las RI entre clientes y proveedores y la diversidad de RI entre empresas del mismo grupo y competidores, dado que son dos categorías en cada grupo, esta será máxima cuando B 
alcance el valor de 0,5 , siendo en este caso $\mathrm{K}=2$. Por último, para la diversidad de tipos de socios en las Rl que se dan entre consultores, universidades, organismos públicos de investigación y centros tecnológicos, dado que $\mathrm{K}=4$, la diversidad máxima será cuando $\mathrm{B}$ alcance el valor de 0,75 .

Para calcular la diversidad mediante este índice se han tomado los datos del Panel que mediante variables dicotómicas permiten identificar si la empresa ha cooperado o no con cada uno de los ocho tipos de socios considerados. Posteriormente se calcularon la diversidad total y la diversidad de los tres grupos. Finalmente, para el análisis del efecto U invertida de la diversidad de RI en la innovación, se han elevado al cuadrado las variables diversidad de RI.

Adicionalmente, hemos incluido cinco variables de control: el tamaño (logaritmo neperiano del número de empleados), la antigüedad de la empresa (número de años que tiene la empresa desde su constitución), el sector (variable dicotómica que toma valor 1 cuando la empresa se dedica a actividades de servicios) y la cuantía dedicada a gastos en I+D internos y externos (porcentaje total de gastos internos y externos en I+D, que es la forma en que la base de datos permite acceder a una referencia del total de gastos de I+D para ambas categorías, y en una escala relativa que permite una comparación más adecuada entre las empresas) (Galende \& Fuente, 2003; Order, Riding, \& Manley, 2006; Pérez-Luño, Cabello-Medina, Carmona-Lavado, \& Cuevas-Rodríguez, 2011).

\section{RESULTADOS Y DISCUSIÓN}

Los estadísticos descriptivos de las 2141 empresas muestran que el 67 \% realiza IP (1433 sí innovan en producto; 708 no), el 56 \% realiza innovación de bienes (1280 sí innovan en bienes; 933 no), el 33 \% realiza innovación de servicios (713 sí innovan en servicios; 1428 no). Por ello, se ha considerado adecuado emplear muestras balanceadas (mismo número de empresas que innovan y no innovan) para el contraste de las hipótesis que permitan dar mayor robustez a los modelos de regresión y evitar errores relacionados con las observaciones atípicas (Chambers, 1986). Así, la muestra para el contraste de la IP son 1416 empresas (708 no innovan en producto; 708 innovan en producto), la muestra para la IB son 1866 empresas (933 no innovan en bienes; 933 innovan en bienes), y la muestra para la IS está formada por 1426 empresas (713 sí innovan en servicios; 713 no innovan en servicios). La Tabla 1 recoge los estadísticos descriptivos, la colinealidad y las correlaciones de cada una de las submuestras balanceadas. En todas ellas las variables de IP, innovación de bien e innovación de servicio se correlacionan significativamente con la variable diversidad de tipos de socios en las RI, y con la diversidad de otro tipo de socios (las RI con consultores, universidades, organismos públicos de investigación y centros tecnológicos), mientras que solo la innovación de bienes además se correlaciona con la diversidad de socios en las relaciones con clientes y proveedores y las relaciones con empresas de mismo grupo y competidores.

Para el contraste de las hipótesis se calcularon 4 modelos de regresión logística binomial (método Wald hacia atrás proporciona una solución con las variables que tienen un mayor nivel de significación) para cada indicador de la variable dependiente (IP, innovación de bienes e innovación de servicios). El modelo 1 permite el contraste de la hipótesis 1 (efecto de la diversidad total en la IP) y el modelo 3 el contraste de las hipótesis 1a, 1 b y $1 \mathrm{c}$ (el efecto de la diversidad de cada uno de los tres grupos en la IP). El modelo 2 permite el contraste de la hipótesis 2 (efecto de U invertida de la diversidad total en la IP) y el modelo 4 el contraste de las hipótesis 2a, 2 b y 2 c (efecto de $U$ invertida de la diversidad de cada uno de los tres grupos en la IP). Aunque las correlaciones de las variables de diversidad de los diferentes grupos y con la diversidad total son bajas en los modelos 2 y 4, se ha optado por separar el contraste en dos modelos de regresión. Las tablas 2, 3 y 4 recogen los resultados de los modelos de regresión logística binomial para la IP (tabla 2), de bienes (tabla 3) y de servicios (tabla 4). 
Tabla 1. Medias, desviaciones típicas, estadísticos de colinealidad y coeficientes de correlación de Pearson

\begin{tabular}{|c|c|c|c|c|c|c|c|c|c|c|c|c|}
\hline \multicolumn{13}{|c|}{ Correlaciones } \\
\hline menta & típica & Hecucticias & & 2 & 3 & 4 & 5 & 6 & 7 & 8 & 9 & 10 \\
\hline
\end{tabular}

Innovación de producto $-\mathrm{N}=1416$

\begin{tabular}{|c|c|c|c|c|c|c|c|c|c|c|c|c|c|}
\hline $\begin{array}{l}\text { 1. Diversidad relaciones- } \\
\text { interorganizacionales }\end{array}$ & 0,360 & 0,302 & & 5,034 & 1 & & & & & & & & \\
\hline $\begin{array}{l}\text { 2. Diversidad Clientes- } \\
\text { Proveedores }\end{array}$ & 0,077 & 0,175 & & 1,673 & $0,271^{* *}$ & 1 & & & & & & & \\
\hline $\begin{array}{l}\text { 3. Diversidad empresas } \\
\text { Mismo Grupo-Competidores }\end{array}$ & 0,037 & 0,123 & & 1,657 & $0,336^{* *}$ & $0,341^{* *}$ & 1 & & & & & & \\
\hline $\begin{array}{l}\text { 4. Diversidad Otros tipos de } \\
\text { socios }\end{array}$ & 0,147 & 0,230 & & 3,254 & $0,656^{* *}$ & $0,174^{* *}$ & $0,314^{* *}$ & 1 & & & & & \\
\hline 5. Tamaño & 4,661 & 1,477 & & 1,226 & $0,165^{* *}$ & $0,122^{*}$ & 0,020 & $0,163^{* *}$ & 1 & & & & \\
\hline 6. Antigüedad & 31,923 & 23,725 & & 1,253 & 0,023 & 0,023 & $-0,037$ & 0,001 & $0,295^{* *}$ & 1 & & & \\
\hline 7. Sector & & & $1=535 / 0=881$ & 1,205 & 0,020 & $-0,104^{*}$ & 0,026 & $0,062^{*}$ & $-0,004$ & $-0,197^{*}$ & 1 & & \\
\hline 8. Gastos internos en I+D & 60,597 & 38,541 & & 1,459 & $0,197^{\star *}$ & 0,006 & $-0,013$ & $0,118^{\star *}$ & $-0,068^{*}$ & $-0,096^{\star \star}$ & $-0,026$ & 1 & \\
\hline 9. Gastos externos en I+D & 13,682 & 23,514 & & 1,416 & 0,015 & 0,067 & 0,020 & $-0,015$ & $0,087^{* *}$ & 0,024 & $-0,064^{*}$ & $-0,327^{\star *}$ & 1 \\
\hline 10. Innovación & & & $1=7080=708$ & & $0,207^{* *}$ & 0,097 & 0,044 & $0,161^{* *}$ & $0,078^{* *}$ & 0,019 & $-0,05$ & $0,180^{* *}$ & $-0,04$ \\
\hline
\end{tabular}

\section{Innovación de bienes $-\mathrm{N}=1866$}

\begin{tabular}{|c|c|c|c|c|c|c|c|c|c|c|c|c|c|c|}
\hline $\begin{array}{l}\text { 1. Diversidad relaciones- } \\
\text { interorganizacionales }\end{array}$ & 0,370 & 0,299 & & 4,802 & 1 & & & & & & & & & \\
\hline $\begin{array}{l}\text { 2. Diversidad Clientes- } \\
\text { Proveedores }\end{array}$ & 0,083 & 0,179 & & 1,587 & $0,291^{* \star}$ & 1 & & & & & & & & \\
\hline $\begin{array}{l}\text { 3. Diversidad empresas } \\
\text { Mismo Grupo-Competidores }\end{array}$ & 0,034 & 0,117 & & 1,533 & $0,322^{* *}$ & $0,293^{* *}$ & 1 & & & & & & & \\
\hline $\begin{array}{l}\text { 4. Diversidad Otros tipos de } \\
\text { socios }\end{array}$ & 0,153 & 0,232 & & 3,185 & $0,654^{* \star}$ & $0,202^{* \star}$ & $0,301^{\star *}$ & 1 & & & & & & \\
\hline 5. Tamaño & 4,713 & 1,514 & & 1,291 & $0,184^{* *}$ & $0,100^{*}$ & 0,036 & $0,180^{* *}$ & 1 & & & & & \\
\hline 6. Antigüedad & 31,887 & 23,259 & & 1,313 & 0,037 & 0,032 & $-0,015$ & 0,022 & $0,326^{* *}$ & 1 & & & & \\
\hline 7. Sector & & & $1=618 / 0=808$ & 1,134 & 0,018 & $-0,051$ & 0,004 & $0,049^{*}$ & $-0,010$ & $-0,205^{\star *}$ & 1 & & & \\
\hline 8. Gastos internos en I+D & 62,625 & 36,804 & & 1,407 & $0,167^{* *}$ & 0,024 & 0,001 & $0,086^{* *}$ & $-0,100^{* *}$ & $-0,113^{*}$ & $-0,013$ & 1 & & \\
\hline 9. Gastos externos en I+D & 13,390 & 22,348 & & 1,333 & 0,032 & 0,032 & 0,001 & 0,002 & $0,068^{* *}$ & $0,046^{*}$ & $-0,064^{\star \star}$ & $-0,329^{\star \star}$ & 1 & \\
\hline 10. Innovación & & & $1=933 / 0=933$ & & $0,190^{* *}$ & $0,120^{\star *}$ & $0,061^{*}$ & $0,140^{* *}$ & $0,076^{* *}$ & 0,034 & $-0,207^{\star *}$ & $0,166^{* *}$ & $-0,006$ & 1 \\
\hline
\end{tabular}

Innovación de servicios - N =1426

\begin{tabular}{|c|c|c|c|c|c|c|c|c|c|c|c|c|c|c|}
\hline $\begin{array}{l}\text { 1. Diversidad relaciones- } \\
\text { interorganizacionales }\end{array}$ & 0,394 & 0,296 & & 4,395 & 1 & & & & & & & & & \\
\hline $\begin{array}{l}\text { 2. Diversidad Clientes- } \\
\text { Proveedores }\end{array}$ & 0,083 & 0,178 & & 1,590 & $0,320^{* *}$ & 1 & & & & & & & & \\
\hline $\begin{array}{l}\text { 3. Diversidad empresas } \\
\text { Mismo Grupo-Competidores }\end{array}$ & 0,038 & 0,123 & & 1,571 & $0,333^{* *}$ & $0,355^{* *}$ & 1 & & & & & & & \\
\hline $\begin{array}{l}\text { 4. Diversidad Otros tipos de } \\
\text { socios }\end{array}$ & 0,163 & 0,238 & & 2,866 & $0,655^{* *}$ & $0,205^{* *}$ & $0,291^{* *}$ & 1 & & & & & & \\
\hline 5. Tamaño & 4,766 & 1,578 & & 1,313 & $0,182^{* *}$ & $0,128^{\star \star}$ & 0,048 & $0,181^{\star \star}$ & 1 & & & & & \\
\hline 6. Antigüedad & 31,256 & 23,606 & & 1,355 & 0,037 & 0,033 & $-0,035$ & 0,007 & $0,356^{* *}$ & 1 & & & & \\
\hline 7. Sector & & & $1=535 / 0=881$ & 1,144 & 0,012 & $-0,021$ & $-0,006$ & $0,069^{*}$ & $-0,029$ & $-0,195^{\star \star}$ & 1 & & & \\
\hline 8. Gastos internos en I+D & 64,737 & 35,302 & & 1,563 & $0,158^{\star \star}$ & $-0,040$ & $-0,008$ & $0,089^{* *}$ & $-0,119^{\star \star}$ & $-0,120^{\star *}$ & $-0,022$ & 1 & & \\
\hline 9. Gastos externos en I+D & 13,099 & 21,642 & & 1,464 & 0,045 & 0,065 & 0,019 & $-0,006$ & $0,059^{*}$ & 0,026 & $-0,044$ & $-0,357^{\star \star}$ & 1 & \\
\hline 10. Innovación & & & $1=713 / 0=713$ & & $0,180^{* *}$ & 0,057 & $-0,031$ & $0,170^{* *}$ & $0,138^{* *}$ & $-0,017$ & $0,269^{* *}$ & $0,092^{* *}$ & $-0,048$ & 1 \\
\hline
\end{tabular}


Tabla 2. Regresiones para la innovación de productos

\begin{tabular}{|c|c|c|c|c|c|c|c|c|c|c|c|c|c|c|c|c|}
\hline \multirow{2}{*}{$\begin{array}{l}\text { Innovación } \\
\text { de producto }\end{array}$} & \multicolumn{4}{|c|}{ Modelo 1} & \multicolumn{4}{|c|}{ Modelo 2} & \multicolumn{4}{|c|}{ Modelo 3} & \multicolumn{4}{|c|}{ Modelo 4} \\
\hline & B & Wald & Sig & $\begin{array}{l}\text { Exp } \\
\text { (B) }\end{array}$ & B & Wald & Sig & $\begin{array}{l}\text { Exp } \\
\text { (B) }\end{array}$ & B & Wald & Sig & $\begin{array}{l}\text { Exp } \\
\text { (B) }\end{array}$ & B & Wald & Sig & $\begin{array}{l}\text { Exp } \\
\text { (B) }\end{array}$ \\
\hline Constante & $-1,477$ & 33,046 & 0,000 & 0,285 & $-1,477$ & 33,046 & 0,000 & 0,285 & $-0,002$ & 0,00 & 0,99 & 0,998 & $-0,76$ & 2,13 & 0,14 & 0,463 \\
\hline Tamaño & 0,088 & 5,295 & 0,021 & 1,092 & 0,088 & 5,295 & 0,021 & 1,092 & 0,095 & 1,12 & 0,29 & 1,099 & 0,138 & 2,83 & 0,09 & 1,148 \\
\hline Antigüedad & 0,000 & 0,008 & 0,928 & 1,000 & 0,000 & 0,003 & 0,955 & 1,000 & $-0,001$ & 0,01 & 0,91 & 0,999 & $-0,00$ & 0,05 & 0,81 & 0,999 \\
\hline Sector & 0,223 & 3,816 & 0,051 & 0,800 & 0,223 & 3,816 & 0,051 & 0,800 & 0,140 & 0,27 & 0,59 & 1,151 & 0,189 & 0,48 & 0,48 & 1,208 \\
\hline $\begin{array}{l}\text { Gastos innovación } \\
\text { interno I+D }\end{array}$ & 0,008 & 31,368 & 0,000 & 1,008 & 0,008 & 31,368 & 0,000 & 1,008 & 0,008 & 5,03 & 0,02 & 1,008 & 0,010 & 6,54 & 0,01 & 1,010 \\
\hline $\begin{array}{l}\text { Gastos innovación } \\
\text { externo I+D }\end{array}$ & , 000 & , 002 & 961 & 1,000 & 0,000 & 0,003 & 0,955 & 1,000 & $-0,006$ & 0,79 & 0,37 & 0,994 & $-0,00$ & 0,79 & 0,37 & 0,994 \\
\hline $\begin{array}{l}\text { Diversidad relaciones- } \\
\text { interorganizacionales }\end{array}$ & 1,169 & 38,299 & 0,000 & 3,219 & 1,169 & 38,299 & 0,000 & 3,219 & & & & & & & & \\
\hline $\begin{array}{l}\text { Diversidad relaciones- } \\
\text { interorganizacionales, } \\
\text { cuadrado }\end{array}$ & & & & & $-0,582$ & 0,355 & 0,551 & 0,559 & & & & & & & & \\
\hline $\begin{array}{l}\text { Diversidad Clientes- } \\
\text { Proveedores }\end{array}$ & & & & & & & & & 0,993 & 1,64 & 0,20 & 2,699 & $-5,68$ & 0,48 & 0,48 & 0,003 \\
\hline $\begin{array}{l}\text { Diversidad empresas } \\
\text { Mismo Grupo- } \\
\text { Competidores }\end{array}$ & & & & & & & & & $-0,535$ & 0,50 & 0,47 & 0,586 & $-8,64$ & 2,87 & 0,09 & 0,000 \\
\hline $\begin{array}{l}\text { Diversidad Otros tipos } \\
\text { de socios }\end{array}$ & & & & & & & & & 0,648 & 1,39 & 0,23 & 1,912 & $-0,21$ & 0,01 & 0,91 & 0,807 \\
\hline $\begin{array}{l}\text { Diversidad Clientes- } \\
\text { Proveedores, } \\
\text { cuadrado }\end{array}$ & & & & & & & & & & & & & 13,69 & 0,64 & 0,42 & 88301 \\
\hline $\begin{array}{l}\text { Diversidad Mismo } \\
\text { Grupo-Competidores, } \\
\text { cuadrado }\end{array}$ & & & & & & & & & & & & & 19,40 & 2,91 & 0,08 & $3 E+08$ \\
\hline $\begin{array}{l}\text { Diversidad Otros tipos } \\
\text { de socios, cuadrado }\end{array}$ & & & & & & & & & & & & & 1,754 & 0,27 & 0,60 & 5,777 \\
\hline -2 Log Verosimilitud & & 1862 & 306 & & & 1862 & 306 & & & & & & & 393 & & \\
\hline R2 Cox-Snell & & 0,0 & & & & 0,0 & & & & & & & & $0, c$ & & \\
\hline R2 Nagelkerke & & 0,0 & & & & 0,0 & & & & & & & & $0, c$ & & \\
\hline$x_{2}$ & & 100, & 687 & & & 100 , & 687 & & & & & & & 10, & & \\
\hline Significación & & 0,0 & oo & & & 0,0 & o० & & & & & & & & & \\
\hline$N$ & & 14 & & & & 14 & & & & & & & & 14 & & \\
\hline $\begin{array}{l}\text { \% Global Tabla } \\
\text { clasificación }\end{array}$ & & 61 & & & & 61 & & & & & & & & 67 & & \\
\hline
\end{tabular}


Tabla 3. Regresiones para la innovación de bienes

\begin{tabular}{|c|c|c|c|c|c|c|c|c|c|c|c|c|c|c|c|c|}
\hline \multirow{2}{*}{$\begin{array}{l}\text { Innovación } \\
\text { de bienes }\end{array}$} & \multicolumn{4}{|c|}{ Modelo 1} & \multicolumn{4}{|c|}{ Modelo 2} & \multicolumn{5}{|c|}{ Modelo 3} & \multicolumn{3}{|c|}{ Modelo 4} \\
\hline & B & Wald & Sig & $\begin{array}{l}\text { Exp } \\
\text { (B) }\end{array}$ & B & Wald & Sig & $\begin{array}{l}\text { Exp } \\
\text { (B) }\end{array}$ & B & Wald & Sig & $\begin{array}{l}\text { Exp } \\
\text { (B) }\end{array}$ & B & Wald & Sig & $\begin{array}{l}\text { Exp } \\
\text { (B) }\end{array}$ \\
\hline Constante & $-1,946$ & 88,23 & 0,000 & 0,143 & $-1,946$ & 88,23 & 0,00 & 0,143 & $-0,464$ & 1,68 & 0,19 & 0,628 & $-0,468$ & 1,712 & 0,19 & 0.626 \\
\hline Tamaño & 0,085 & 6,524 & 0,011 & 1,089 & 0,085 & 6,524 & 0,01 & 1,089 & 0,111 & 2,70 & 0,10 & 1,118 & 0,113 & 2,792 & 0,09 & 1.119 \\
\hline Antigüedad & $-0,002$ & 0,726 & 0,394 & 0,998 & $-0,002$ & 0,759 & 0,38 & 0,998 & 0,002 & 0,12 & 0,72 & 1,002 & 0,002 & 0,115 & 0,73 & 1.002 \\
\hline Sector & 0,926 & 83,71 & 0,000 & 2,524 & 0,926 & 83,71 & 0,00 & 2,524 & 0,516 & 6,07 & 0,01 & 1,675 & 0,511 & 5,948 & 0,01 & 1.666 \\
\hline $\begin{array}{l}\text { Gastos innovación } \\
\text { interno I+D }\end{array}$ & 0,009 & 38,94 & 0,000 & 1,009 & 0,009 & 38,94 & 0,00 & 1,009 & 0,002 & 0,39 & 0,52 & 1,002 & 0,003 & 0,500 & 0,47 & 1.003 \\
\hline $\begin{array}{l}\text { Gastos innovación } \\
\text { externo I+D }\end{array}$ & 0,002 & 0,981 & 0,322 & 1,002 & 0,002 & 0,963 & 0,32 & 1,002 & $-0,003$ & 0,34 & 0,55 & 0,997 & $-0,003$ & 0,316 & 0,57 & 0.997 \\
\hline $\begin{array}{l}\text { Diversidad relaciones- } \\
\text { interorganizacionales }\end{array}$ & 1,167 & 47,28 & 0,000 & 3,214 & 1,167 & 47,28 & 0,00 & 3,214 & & & & & & & & \\
\hline $\begin{array}{l}\text { Diversidad relaciones- } \\
\text { interorganizacionales, } \\
\text { cuadrado }\end{array}$ & & & & & $-0,619$ & 0,525 & 0,46 & 0,538 & & & & & & & & \\
\hline $\begin{array}{l}\text { Diversidad Clientes- } \\
\text { Proveedores }\end{array}$ & & & & & & & & & 1,308 & 4,77 & 0,02 & 3,700 & 0,077 & 0,000 & 0,99 & 1.080 \\
\hline $\begin{array}{l}\text { Diversidad empresas } \\
\text { Mismo Grupo- } \\
\text { Competidores }\end{array}$ & & & & & & & & & $-0,278$ & 0,17 & 0,67 & 0,757 & $-4,208$ & 0,865 & 0,35 & 0.015 \\
\hline $\begin{array}{l}\text { Diversidad Otros tipos } \\
\text { de socios }\end{array}$ & & & & & & & & & 0,554 & 1,35 & 0,24 & 1,740 & 0,537 & 0,107 & 0,74 & 1.710 \\
\hline $\begin{array}{l}\text { Diversidad Clientes- } \\
\text { Proveedores, } \\
\text { cuadrado }\end{array}$ & & & & & & & & & & & & & 2,782 & 4,800 & 0,02 & 16.154 \\
\hline $\begin{array}{l}\text { Diversidad Mismo } \\
\text { Grupo-Competidores, } \\
\text { cuadrado }\end{array}$ & & & & & & & & & & & & & 8,877 & 0,763 & 0,38 & 7167 \\
\hline $\begin{array}{l}\text { Diversidad Otros tipos } \\
\text { de socios, cuadrado }\end{array}$ & & & & & & & & & & & & & 0,102 & 0,001 & 0,97 & 1.107 \\
\hline -2 Log Verosimilitud & & 2388 & 3,900 & & & 2388 & 900 & & & & 533,882 & & & 533 & & \\
\hline R2 Cox-Snell & & 0, & 101 & & & 0, & & & & & 0,041 & & & & & \\
\hline R2 Nagelkerke & & 0, & 134 & & & 0, & & & & & 0,056 & & & & & \\
\hline$x_{2}$ & & 197, & 926 & & & 197, & & & & & 17,550 & & & & & \\
\hline Significación & & 0,0 & boo & & & & & & & & 0,001 & & & & & \\
\hline $\mathrm{N}$ & & 18 & 66 & & & 18 & & & & & 1866 & & & 18 & & \\
\hline $\begin{array}{l}\text { \% Global Tabla } \\
\text { clasificación }\end{array}$ & & 62 & 2,5 & & & 62 & & & & & 60,8 & & & & & \\
\hline
\end{tabular}


Tabla 4. Regresiones para la innovación en servicios

\begin{tabular}{|c|c|c|c|c|c|c|c|c|c|c|c|c|c|c|c|c|}
\hline \multirow{2}{*}{$\begin{array}{l}\text { Innovación } \\
\text { de servicios }\end{array}$} & \multicolumn{4}{|c|}{ Modelo 1} & \multicolumn{4}{|c|}{ Modelo 2} & \multicolumn{4}{|c|}{ Modelo 3} & \multicolumn{4}{|c|}{ Modelo 4} \\
\hline & B & Wald & Sig & $\begin{array}{l}\text { Exp } \\
\text { (B) }\end{array}$ & B & Wald & Sig & $\begin{array}{l}\text { Exp } \\
\text { (B) }\end{array}$ & B & Wald & Sig & $\begin{array}{l}\text { Exp } \\
\text { (B) }\end{array}$ & B & Wald & Sig & $\begin{array}{l}\text { Exp } \\
\text { (B) }\end{array}$ \\
\hline Constante & $-1,04$ & 20,214 & 0,00 & 0,353 & $|-0,994|$ & 18,539 & 0,00 & 0,370 & $-0,650$ & 2,371 & 0,12 & 0,522 & $-0,556$ & 1,783 & 0,18 & 0,573 \\
\hline Tamaño & 0,195 & 25,863 & 0,00 & 1,215 & 0,190 & 24,457 & 0,00 & 1,209 & 0,266 & 11,372 & 0,00 & 1,305 & 0,266 & 11,24 & 0,00 & 1,304 \\
\hline Antigüedad & 0,000 & 0,033 & 0,85 & 1,000 & 0,000 & 0,019 & 0,88 & 1,000 & 0,001 & 0,027 & 0,87 & 1,001 & 0,001 & 0,011 & 0,91 & 1,001 \\
\hline Sector & $-1,20$ & 107,98 & 0,00 & 0,299 & $-1,194$ & 105,39 & 0,00 & 0,303 & $-1,125$ & 21,150 & 0,00 & 0,325 & $-1,116$ & 20,70 & 0,00 & 0,327 \\
\hline $\begin{array}{l}\text { Gastos innovación } \\
\text { interno I+D }\end{array}$ & 0,006 & 12,847 & 0,00 & 1,006 & 0,006 & 13,364 & 0,00 & 1,006 & 0,005 & 1,240 & 0,26 & 1,005 & 0,006 & 1,465 & 0,22 & 1,006 \\
\hline $\begin{array}{l}\text { Gastos innovación } \\
\text { externo I+D }\end{array}$ & $-0,00$ & 0,622 & 0,43 & 0,998 & $-0,002$ & 0,639 & 0,42 & 0,998 & 0,000 & 0,000 & 0,99 & 1,000 & 0,001 & 0,007 & 0,93 & 1,001 \\
\hline $\begin{array}{l}\text { Diversidad relaciones- } \\
\text { interorganizacionales }\end{array}$ & 1,050 & 27,856 & , 000 & 2,858 & $-0,029$ & 0,002 & 0,96 & 0,971 & & & & & & & & \\
\hline $\begin{array}{l}\text { Diversidad relaciones- } \\
\text { interorganizacionales, } \\
\text { cuadrado }\end{array}$ & & & & & 1,526 & 30,409 & 0,00 & 4,600 & & & & & & & & \\
\hline $\begin{array}{l}\text { Diversidad Clientes- } \\
\text { Proveedores }\end{array}$ & & & & & & & & & 0,206 & 0,091 & 0,76 & 1,228 & $-4,048$ & 0,461 & 0,49 & 0,017 \\
\hline $\begin{array}{l}\text { Diversidad empresas } \\
\text { Mismo Grupo- } \\
\text { Competidores }\end{array}$ & & & & & & & & & $-1,065$ & 2,183 & 0,14 & 0,345 & $-1,184$ & 2,985 & 0,08 & 0,306 \\
\hline $\begin{array}{l}\text { Diversidad Otros tipos } \\
\text { de socios }\end{array}$ & & & & & & & & & 0,957 & 3,6690 & 0,05 & 2,604 & 0,145 & 0,007 & 0,93 & 1,156 \\
\hline $\begin{array}{l}\text { Diversidad Clientes- } \\
\text { Proveedores, cuadrado }\end{array}$ & & & & & & & & & & & & & 8,974 & 0,510 & 0,47 & 7894,31 \\
\hline $\begin{array}{l}\text { Diversidad Mismo Grupo- } \\
\text { Competidores, cuadrado }\end{array}$ & & & & & & & & & & & & & 7,584 & 0,479 & 0,48 & 1966,12 \\
\hline $\begin{array}{l}\text { Diversidad Otros tipos de } \\
\text { socios, cuadrado }\end{array}$ & & & & & & & & & & & & & 1,912 & 4,804 & 0,02 & 6,770 \\
\hline -2 Log Verosimilitud & & 1788 & .757 & & & 1785 & 982 & & & 437, & & & & & 949 & \\
\hline R2 Cox-Snell & & 0,1 & & & & 0,1 & & & & 0,1 & & & & & 114 & \\
\hline R2 Nagelkerke & & 0,1 & & & & 0,1 & & & & 0,1 & & & & & 154 & \\
\hline$x_{2}$ & & 188, & & & & 190 , & & & & 40,2 & & & & & 077 & \\
\hline Significación & & 0,0 & & & & 0,0 & & & & 0,0 & & & & & poo & \\
\hline $\mathrm{N}$ & & 14 & & & & 14 & & & & 142 & & & & & 26 & \\
\hline $\begin{array}{l}\text { \% Global Tabla } \\
\text { clasificación }\end{array}$ & & 65 & 4 & & & 64 & & & & 65 & & & & & 5,7 & \\
\hline
\end{tabular}


A pesar de la esperada colinealidad entre las variables y sus valores cuadráticos para analizar el efecto de $\mathrm{U}$ invertida, los parámetros de ajuste de los modelos 1 y 2 de las tablas 2 y 3 para el caso de la variable diversidad de RI no han cambiado. Los modelos 3 y 4 de las tablas 2, 3 y 4 ofrecen un mejor ajuste, son más consistentes para relacionar las variables de estudio (-2 Log Verosimilitud, R2 Cox-Snell, R2 Nagelkerke, $X^{2}$ ).

Respecto a la hipótesis 1 (influencia positiva de la diversidad de las RI en la innovación), los resultados de los modelos para la IP, IB e IS (modelos 1) permiten concluir que esta hipótesis se confirma de forma positiva y con un nivel de confianza del $99 \%$. Estos resultados muestran que la diversidad de tipos de socios en las RI hace 3,219 veces más probable la IP, 3,214 veces la IB, y 2,858 veces la IS. Estos resultados son consistentes con el trabajo de Leeuw et al. (2014) quienes encontraron una relación positiva y significativa entre la diversidad de Rl y la innovación.

Para las hipótesis 1a, 1b y 1c (efecto de la diversidad de socios en las RI en la innovación por cada uno de los tres grupos de tipos de socios con los que interactúa la empresa), los resultados de los modelos de regresión 3 en la tabla 3 nos permiten confirmar parcialmente la hipótesis 1a (la diversidad de clientes y proveedores favorece la IB) de forma positiva y con un nivel de confianza del $97 \%$, indicando que hace 3,700 veces más probable la IB. Este resultado es consistente con Ozer y Zhang (2015) que confirman que las redes con proveedores y clientes potencian la IP.

La hipótesis 1c en la tabla 4 (la diversidad de consultores, universidades, organismos públicos de investigación y centros tecnológicos favorece la innovación de servicios) se confirma de forma positiva con un nivel de confianza del $95 \%$, haciendo más probable la IS 2,604 veces. Este tipo de diversidad de relaciones permite a las empresas acceder a variados recursos y conocimientos necesarios para la innovación de bienes y servicios (Wassmer, Li, \& Madhok, 2017). Sin embargo, contrario a resultados previos, los resultados no muestran un efecto significativo para la hipótesis 1 b, las empresas no obtienen un efecto positivo sobre la IP derivada de la diversidad de las RI con empresas del mismo grupo y con competidores.

Respecto a la hipótesis 2 (relación en forma de $U$ invertida entre la diversidad de tipos de socios y la innovación), los resultados obtenidos no confirman esta hipótesis. Los resultados ponen de manifiesto que la relación entre diversidad de socios en las Rl y la innovación sigue una relación lineal, a diferencia del trabajo de Hagedoorn et al. (2018) y Hottenrott y Lopes-Bento (2016) quienes demostraron que existe una relación en forma de U invertida entre estas variables. Una posible explicación a este resultado es que los vínculos de la red aumentan la confianza y las relaciones interpersonales entre los socios, lo cual ayuda a mantener el efecto positivo de la diversidad de socios en la innovación. Además, cuanto más arraigados están esos vínculos, mayor es la confianza y reciprocidad entre los socios (Burt, 2004; Mariotti \& Delbridge, 2012; Ozer \& Zhang, 2015; Uzzi, 1997; Uzzi \& Lancaster, 2003). En cualquier caso, este resultado requiere un análisis más profundo en investigaciones futuras.

Por último, con relación a las hipótesis $2 \mathrm{a}, 2 \mathrm{~b}$ y 2 c que proponían el efecto en forma de $U$ invertida en la relación diversidad de RI y la innovación en cada uno de los tres grupos de socios estratégicos, los resultados de los modelos 4 no permiten confirmar estas hipótesis para la innovación de bienes y la innovación de servicios. Así, en cuanto a la hipótesis za (el efecto de la diversidad de RI con clientes y proveedores en la IP sigue una relación en forma de $U$ invertida), en el modelo 4 de la tabla 3 el coeficiente de regresión es positivo y significativo, indicando que la relación entre diversidad de relaciones de clientes y proveedores y la innovación de bienes sigue una relación lineal. Para la hipótesis 2 c (el efecto de la diversidad de RI con consultores, universidades, organismos públicos de investigación y centros tecnológicos en la IP sigue una relación en forma de U invertida), en el modelo 4 de la tabla 4 el coeficiente de regresión es positivo y significativo, indicando que la 
relación entre la diversidad de relaciones con consultores, universidades y entidades de investigación y la innovación de servicios sigue una relación lineal (sin punto de inflexión), haciendo más probable la innovación en 6,770 veces. Estos resultados no confirman que puede surgir una alta sensibilidad y desconfianza entre empresas al incrementar la diversidad de Rl según Kobarg et al. (2019).

En cuanto a las variables de control, el tamaño de la empresa es un factor que afecta significativamente a las actividades de innovación, debido a que influye positivamente en la capacidad de absorción, promoviendo en mayor medida las actividades de innovación (Beck \& Schender-Wicki, 2014). En relación al sector, este tiene una influencia positiva y significativa en la innovación de bienes (Østergaard, Timmermans, \& Kristinsson, 2011). En cuanto a los gastos internos en I+D, los resultados muestran que son de importancia para las innovaciones de productos, ya sea de bienes o servicios. Los modelos indican que la variable antigüedad y gastos de innovación externos en I+D no tienen un impacto significativa.

\section{CONCLUSIONES}

Teniendo en cuenta la importancia de las RI en la IP (Mayskens \& Carssrud, 2013), este trabajo aporta nueva evidencia en el estudio de la influencia de la diversidad de RI en la IP, ya sea de bienes o de servicios. Asimismo, hemos analizado si el efecto de la diversidad de tipos de socios en las RI en la IP varía según el tipo de socio estratégico con el que la empresa decida relacionarse.

Con los datos del Panel de Innovación Tecnológica de España se desarrollaron 12 modelos de regresión logística binomial que permitieron mostrar el efecto positivo y significativo de la diversidad de socios en las RI en la IP, tanto de bienes como servicios (Beck \& Schenker-Wicki, 2014). Cuanto más diversos sean los tipos de socios estratégicos con los que la empresa decida relacionarse, mayor será la probabilidad de obtener conocimientos heterogéneos y complementarios relevantes para promover las innovaciones de producto (Amara \& Landry, 2005; Leeuw et al., 2014; Meyskens \& Carssrud, 2013; Nieto \& Santamaría, 2007). Este trabajo muestra que la diversidad de relaciones que la empresa mantiene con socios comerciales (clientes y proveedores) favorece más la innovación de bienes, al acceder a conocimientos relacionados con el mercado, necesarios para desarrollar innovaciones de bienes e introducirlas con éxito en el mercado (Arranz \& Arroyabe, 2008). Las RI con clientes y proveedores tiene un papel crucial en las actividades de I+D porque contribuyen con conocimientos complementarios relacionados con las necesidades de los clientes (Arranz \& Arroyabe, 2008). Igualmente, la diversidad de relaciones que la empresa mantiene con socios no industriales (consultores, universidades y entidades de investigación) favorece los beneficios de la diversidad en la innovación de servicios. Este tipo de relaciones permite acceder a conocimientos tecnológicos para el desarrollo de servicios novedosos conforme con las nuevas tecnologías. Este resultado coincide con trabajos previos que sostienen que las relaciones con universidades abren nuevos segmentos de mercado favoreciendo la IP, al acceder a nuevos conocimientos necesarios para llevar a cabo innovaciones de servicios, de alta calidad y novedosas (Laursen \& Salter, 2006; Tether, 2002) y favorecen la comercialización de las innovaciones (Oerlemans, Knoben, \& Pretorius, 2013). Resumiendo, este trabajo permite demostrar que la diversidad de RI es beneficiosa para la IP, ya sea de bienes o servicios (Wassmer et al., 2017). La diversidad de relaciones con clientes y proveedores potencia los beneficios de la diversidad en la innovación de bienes; y los consultores, universidades y entidades de investigación, en la innovación de servicios. Finalmente, los resultados no permiten afirmar que demasiada diversidad produzca rendimientos decrecientes 
en la innovación. La diversidad (global y por tipos de socios) provee a la empresa de conocimientos moderadamente relacionados y complementarios. En nuestra muestra la mayor diversidad de RI es valiosa sin perjudicar su efecto en la IP (Noseleit \& Faria, 2013).

Los resultados de este estudio tienen implicaciones prácticas, tanto a nivel gubernamental como a nivel empresarial. A nivel gubernamental, es de relevancia que el gobierno español haga uso de incentivos para fomentar la IP que se derivan del aprovechamiento de los conocimientos externos adquiridos de las RI, ya que muchas empresas no tienen los conocimientos internos y los recursos suficientes para innovar y mantenerse competitivas (Beers \& Zand, 2014). Por lo tanto, necesitan de las relaciones con otras empresas para incrementar las ventas, mejorar la competitividad y por ende mantenerse por mucho más tiempo en el mercado a través de las innovaciones de productos.

Los resultados de este estudio tienen implicaciones prácticas, tanto a nivel gubernamental, como a nivel empresarial. Los resultados muestran la importancia y necesidad de promover el desarrollo de políticas que fomenten las relaciones interorganizacionales. A nivel empresarial, es importante que la empresa defina políticas de gestión del conocimiento que faciliten la apropiación de los conocimientos externos provenientes de dichas relaciones interorganizacionales y que, al mismo tiempo, disminuyan el riesgo de fuga de conocimientos clave para mantener la ventaja competitiva.

En todo caso, los resultados están limitados por la fuente de información empleada. Por ello, futuras investigaciones deben utilizar otros contextos empíricos, así como estudiar la influencia de las políticas gubernamentales o aspectos culturales, otras formas de innovación (incremental y radical) o analizar si hay diferencias en función de las características de las empresas, así como profundizar en la relación en U invertida que en este estudio no se ha podido confirmar.

\section{REFERENCIAS}

Amara, N., \& Landry, R. (2005). Sources of information as determinants of novelty of innovation in manufacturing firms: Evidence from the 1999 Statistics Canada Innovation Survey. Technovation, 25(3), 145-259. doi:10.1016/S01664972(03)00113-5

Ardito, L., Petruzzelli, A. M., \& Albino, V. (2015). From technological inventions to new products: A systematic review and research agenda of the main enabling factors. European Management Review, 12(3), 113-147. doi: 10.1111/emre.12047

Arranz, N., \& Arroyabe, J. C. F. (2008). The choice of partners in R\&D cooperation: An empirical analysis of Spanish firms. Technovation, 28(1), 88-100. doi: 10.1016/j. technovation.2007.07.006

Ashok, M., Narula, R., \& Martínez-Noya, A. (2016). How do collaboration and investments in knowledge management affect process innovation in services? Journal of Knowledge Management, 20(5), 1004-1024. doi: 10.1108/JKM-11-20150429

Beck, M., \& Schenker-Wicki, A. (2014). Cooperating with external partners: The importance of diversity for innovation performance. European Journal of International Management, 8(5), 548-569. doi:10.1504/EJIM.2014.064604
Beers, C. V., \& Zand, F. (2014). R\&D cooperation, partner diversity, and innovation performance: An empirical analysis. Journal Production Innovation Management, 31(2), 292-312. doi:10.1111/jpim.12096

Belderbos, R., Carree, M., \& Lokshin, B. (2004). Co-operative R\&D and firm performance. Research Policy, 33(10), 14771492. doi: 10.1016/j.respol.2004.07.003

Belderbos, R., Carree, M., Lokshin, B., \& Sastre, J. F. (2015). Inter-temporal patterns of R\&D collaboration and innovative performance. The Journal of Technology Transfer, 40(1), 123137. doi: 10.1007/s10961-014-9332-4

Belderbos, R., Gilsing, V., Lokshin, B., Carree, M., \& Sastre, J. F. (2018). The antecedents of new R\&D collaborations with different partner types: On the dynamics of past R\&D collaboration and innovative performance. Long Range Planning, 51(2), 285-302. doi: 10.1016/j.lrp.2017.10.002

Bessant, J., \& Rush, H. (1995). Building bridges for innovation: The role of consultants in technology transfer. Research Policy, 24(1), 97-114. doi: 10.1016/0048-7333(93)00751-E

Bogers, M., Afuah, A., \& Bastian, B. (2010). Users as innovators: A review, critique, and future research directions. Journal of Management, 36(4), 857-875. doi: 10.1177/0149206309353944V 
Bruce, M., Leverick, F., Littler, D., \& Wilson, D. (1995). Success factors for collaborative product development: A study of suppliers of information and communication technology. $R \& D$ Management, 25(1), 33-44. doi: 10.1111/j.1467-9310.1995 tboo898.x

Burg, E., Berends, H., \& Raaij, E. M. Van. (2014). Framing and interorganizational knowledge transfer: A process study of collaborative innovation in the aircraft industry. Journal of Management Studies, 51(3), 349-378. doi: 10.1111/joms.12055

Burt, R. S. (2004). Structural holes and good ideas. American Journal of Sociology, 110(2), 349-399. doi: 10.1086/421787

Chambers, R. L. (1986). Outlier robust finite population estimation. Journal of the American Statistical Association, 81(396), 1063-1069. doi: 10.1080/01621459.1986.10478374

Chen, J., Chen, Y. F., \& Vanhaverbeke, W. (2011). The influence of scope, depth, and orientation of external technology sources on the innovative performance of Chinese firms. Technovation, 31(8), 362-373. doi: 10.1016/j.technovation.2011.03.002

Das, T., \& Teng, B. (2000). A resource-based theory of strategic alliances. Journal of Management, 26(1), 31-36. doi: 10.1016/ S0149-2063(99)00037-9

Dowling, M., \& Helm, R. (2006). Product development success through cooperation: A study of entrepreneurial firms. Technovation, 26(4), 483-488. doi: 10.1016/j. technovation.2005.06.015

Dussauge, P., Garrette, B., \& Mitchell, W. (2000). Learning from competing partners: Outcomes and durations of scale and link alliances in Europe, North America and Asia. Strategic Management Journal, 21(2), 99-126. doi: 10.1002/(SICI)10970266(200002)21:2〈99::AID-SMJ80>3.0.CO;2-G

Duysters, G., \& Lokshin, B. (2011). Determinants of alliance portfolio complexity and its effect on innovative performance of companies. Journal of Product Innovation Management, 28(4), 570-585. doi: 10.1111/j.1540-5885.2011.00824.X

Emden, Z., Calantone, R. J., \& Droge, C. (2006). Collaborating for new product development: Selecting the partner with maximum potential to create value. Journal of Product Innovation Management, 23(4), 330-341. doi: 10.1111/j.1540$5885.2006 .00205 . x$

Faems, D., Janssens, M., Madhok, A., \& Looy, B. Van. (2008) Towards an integrative perspective on alliance governance: Connecting contract design, contract application and trust dynamics. Academy of Management Journal, 51(6), 1053-1078. doi: 10.5465/amj.2008.35732527

Faems, D., Looy, B.Van, \& Debackere, K. (2005). Interorganizational collaboration and innovation: Toward a portfolio approach. Journal of Product Innovation Management, 22(3), 238-250. doi: 10.1111/j.0737-6782.2005.00120.x

Frenz, M., \& letto-Gillies, G. (2009). The impact on innovation performance of different sources of knowledge: Evidence from the UK Community Innovation Survey. Research Policy, 38(7), 1125-1135. doi: 10.1016/j.respol.2009.05.002

Galende, J., \& Fuente, J. De la. (2003). Internal factors determining a firm's innovative behaviour. Research Policy, 32(5), 715-736. doi:10.1016/S0048-7333(02)00082-3

Gimeno, J. (2004). Competition within and between networks: The contingent effect of competitive embeddedness on alliance formation. Academy of Management Journal, 47(6), 820-842. doi: $10.5465 / 20159625$
Haans, R. F., Pieters, C., \& Zi-Lin, H. (2016). Thinking about U: Theorizing and testing $\mathrm{U}$ - and inverted $\mathrm{U}$-shaped relationships in strategy research. Strategic Management Journal, 37(7), 1177-1195. doi: 10.1002/smj.2399

Hagedoorn, J., Lokshin, B., \& Zobel, A. K. (2018). Partner type diversity in alliance portfolios: Multiple dimensions, boundary conditions and firm innovation performance. Journal of Management Studies, 55(5), 809-836. doi: 10.1111/ joms.12326

Hottenrott, H., \& Lopes-Bento, C. (2016). R\&D partnerships and innovation performance: Can there be too much of a good thing?. Journal of Product Innovation Management, 33, 773794. doi: 10.1111/jpim.12311

Jiang, R. J., Qingjiu, T. T., \& Santoro, M. D. (2010). Alliance portfolio diversity and firm performance. Strategic Management Journal, 31(10), 1136-1144. doi: 10.1002/smj.869

Knudsen, M. P., \& Mortensen, T. B. (2011). Some immediate but negative - effects of openness on product development performance. Technovation, 31(1), 54-64. doi: 10.1016/j. technovation.2010.07.002

Kobarg, S., Stumpf-Wollersheim, J., \& Welpe, I. M. (2019). More is not always better: Effects of collaboration breadth and depth on radical and incremental innovation performance at the project level. Research Policy, 48(1), 1-10. doi: 10.1016/j. respol.2018.07.014

Laursen, K., \& Salter, A. (2006). Open for innovation: The role of openness in explaining innovation performance among UK manufacturing firms. Strategic Management Journal, 27(2), 131-150. doi: 10.1002/smj.507

Lavie, D. (2006). The competitive advantage of interconnected firms: An extension of the resource-based view. Academy of Management Review, 31(3), 638-658. doi: 10.5465/ amr.2006.21318922v

Lavie, D. (2009). Capturing value from alliance portfolios. Organizational Dynamics, 38(1), 26-36. doi: 10.1016/j. orgdyn.2008.04.008

Ledwith, A., \& Coughlan, P. (2005). Splendid isolation: Does networking really increase new product success? Creativity and Innovation Management, 14(4), 366-373. doi: 10.1111/j.1467-8691.2005.00356.x

Lee, D., Kirkpatrick-Husk, K., \& Madhavan, R. (2017). Diversity in alliance portfolios and performance outcomes: A metaanalysis. Journal of Management, 43(5), 1472-1497. doi: $10.1177 / 0149206314556316$

Leeuw, T. De, Lokshin, B., \& Duysters, G. (2014). Returns to alliance portfolio diversity: The relative effects of partner diversity on firm's innovative performance and productivity. Journal of Business Research, 67(9), 1839-1849. doi: 10.1016/j. jbusres.2013.12.005

Luo, Y. (2002). Contract, cooperation and performance in international joint ventures. Strategic Management Journal, 23(10), 903-919. doi: 10.1002/smj.261V

Mariotti, F., \& Delbridge, R. (2012). Overcoming network overload and redundancy in interorganizational networks: The roles of potential and latent ties. Organization Science, 23(2), 511-528. doi: 10.1287 /orsc.1100.0634

Martinez-Noya, A., \& Narula, R. (2018). What more can we learn from R\&D alliances? A review and research agenda. $B R Q$ Business Research Quarterly, 21(3), 195-212. doi: 10.1016/j. brq.2018.04.001 
Meyskens, M., \& Carsrud, A. L. (2013). Nascent green-technology ventures: A study assessing the role of partnership diversity in firm success. Small Business Economics, 40(3), 739-759. doi: $10.1007 /$ S11187-011-9400-1

Miotti, L., \& Sachwald, F. (2003). Co-operative R\&D: why, and with whom? An integrated framework of analysis. Research Policy, 32(8), 1481-1499. doi: 10.1016/So048-7333(02)001592

Monteiro, F., Mol, M., \& Birkinshaw, J. (2017). Ready to be open? Explaining the firm level barriers to benefiting from openness to external knowledge. Long Range Plan, 50(2), 282-295. doi: 10.1016/j.Irp.2015.12.008

Mowery, D. C., Oxley, J. E., \& Silverman, B. S. (1996). Strategic alliances and interfirm knowledge transfer. Strategic Management Journal, 17(S2), 77-91. doi: 10.1002/ smj.4250171108

Nieto, M., \& Santamaría, L. (2010). Colaboración tecnológica e innovación en las empresas de base tecnológica: Implicaciones de las relaciones con universidades y otros socios tecnológicos. Revista Galega de Economía, 19, 11322799. https://www.redalyc.org/pdf/391/39115737009.pdf

Nieto, M. J., \& Santamaría, L. (2007). The importance of diverse collaborative networks for the novelty of product innovation. Technovation, 27(3), 367-377. doi: 10.1016/j. technovation.2006.10.001

Noseleit, F., \& Faria, P. de. (2013). Complementarities of internal R\&D and alliances with different partner types. Journal of Business Research, 66(10), 2000-2006. doi: 10.1016/j. jbusres.2013.02.025V

Numprasertchai, S., \& Igel, B. (2005). Managing knowledge through collaboration: Multiple case studies of managing research in university laboratories in Thailand. Technovation, 25(10), 1173-1182. doi: 10.1016/j.technovation.2004.03.001

Oerlemans, L. A. G., Knoben, J., \& Pretorius, M. W. (2013). Alliance portfolio diversity, radical and incremental innovation: The moderating role of technology management. Technovation, 33(6), 234-246. doi: 10.1016/j.technovation.2013.02.004

Order, B., Riding, A., \& Manley, K. (2006). Women entrepreneurs and financial capital. Entrepreneurship Theory and Practice, 30(5), 643-665. doi: 10.1111/j.1540-6520.2006.00140.x

Østergaard, C. R., Timmermans, B., \& Kristinsson, K. (2011). Does a different view create something new? The effect of employee diversity on innovation. Research Policy, 40(3), 500-509. doi: 10.1016/j.respol.2010.11.004

Ozer, M., \& Zhang, W. (2015). The effects of geographic and network ties on exploitative and exploratory product innovation. Strategic Management Journal, 36(7), 1105-1114. doi: $10.1002 / \mathrm{smj} .2263$

Pérez-Luño, A., Cabello-Medina, C., Carmona-Lavado, A., \& Cuevas-Rodríguez, G. (2011). How social capital and knowledge affect innovation. Journal of Business Research, 64(2), 1369-1376. doi: 10.1016/j.jbusres.2011.01.014
Romijn, H., \& Albadalejo, M. (2002). Determinants of innovation capability in small electronics and software firms in southeast England. Research Policy, 31(7), 1053-1067. doi: 10.1016/ So048-7333(01)00176-7

Rosenzweig, E. D., Roth, A. V., \& Dean, J. W. (2003). The influence of an integration strategy on competitive capabilities and business performance: An exploratory study of consumer products manufacturers. Journal of Operations Management, 21(4), 437-456. doi: 10.1016/So272-6963(03)00037-8

Santamaría, L., Nieto, M. J., \& Miles, I. (2012). Service innovation in manufacturing firms: Evidence from Spain. Technovation, 32(2), 144-155. doi: 10.1016/j.technovation.2011.08.006

Schoenmakers, W., \& Duysters, G. (2006). Learning in strategic technology alliances. Technology Analysis \& Strategic Management, 18(2), 245-264. doi: 10.1080/09537320600624162

Silva, M. J., \& Leitão, J. (2009). Cooperation in innovation practices among firms in Portugal: Do external partners stimulate innovative advances? International Journal of Entrepreneurship and Small Business, 7(4), 391-403. doi: 10.1504/IJESB.2009.023357

Simonen, J., \& McCann, P. (2008). Innovation, R\&D cooperation and labor recruitment: evidence from Finland. Small Business Economics, 31(2), 181-194. doi: 10.1007/s11187-007-9089-3

Story, V., O'Malley, \& L., Hart, S. (2011). Roles, role performance, and radical innovation competences. Industrial Marketing Management, 40(6), 952-966. doi: 10.1016/j. indmarman.2011.06.025

Tether, B. (2002). Who cooperate for innovation, and why: And empirical analysis. Research Policy, 31(6), 947-967. doi: 10.1016/S0048-7333(01)00172-X

Uzzi, B. (1997). Social structure and competition in interfirm networks: The paradox of embeddedness. Administrative Science Quarterly, 42(1), 35-67. doi: 10.2307/2393808

Uzzi, B., \& Lancaster, R. (2003). Relational embeddedness and learning: The case of bank loan managers and their clients. Management Science, 49(4), 383-399. doi: 10.1287/ mnsc.49.4.383.14427

Vasudeva, G., \& Anand, J. (2011). Unpacking absorptive capacity: A study of knowledge utilization from alliance portfolios. Academy of Management Journal, 54(3), 611-623. doi: 10.5465/amj.2011.61968108v

Wagner, S. M., \& Hoegl, M. (2006). Involving suppliers in product development: Insights from R\&D directors and project managers. Industrial Marketing Management, 35(8), 936-943. doi: 10.1016/j.indmarman.2005.10.009v

Wassmer, U., Li, S., \& Madhok, A. (2017). Resource ambidexterity through alliance portfolios and firm performance. Strategic Management Journal, 38(2), 384-394. doi: 10.1002/smj.2488

Wuyts, S., \& Dutta, S. (2014). Benefiting from alliance portfolio diversity: The role of past internal knowledge creation strategy. Journal of Management, 40(6), 1653-1674. doi: $10.1177 / 0149206312442339 \mathrm{~V}$

\section{CONTRIBUCIÓN DE LOS AUTORES}

Los autores declaran haber participado en todas las etapas de desarrollo del manuscrito. Todos los autores trabajaron juntos en todas las secciones, la redacción y revisión final del manuscrito. 